\title{
El gaélico irlandés en Bizkaia durante la Edad Moderna
}

\section{Ekain Cagigal Montalbán}

Durante la Edad Moderna, el Señorío de Bizkaia acogió una pequeña comunidad de refugiados católicos irlandeses, que, huyendo de la represión protestante de Irlanda, encontró diferentes vías de progreso, principalmente, ligado al apogeo del comercio atlántico. Tanto en el seno de este colectivo como en espacios con vocación más públi$\mathrm{ca}$, asociado a las actividades mercantiles y portuarias de Bizkaia, la lengua irlandesa jugó su papel en diferentes dominios del ámbito socio-económico: autos del Consulado de Bilbao, causas judiciales, procesos de avecindamiento de irlandeses, actividad artesanal de los talleres de curtido, los propios espacios de sociabilidad habituales de los vizcaínos, o dentro de las propias familias de emigrados. En todos ellos, e indudablemente, en diferentes formas, el gaélico irlandés encontró su espacio como un modo de reivindicación y pervivencia de los rasgos identitarios de una sociedad en el exilio.

Palabras claves: Gaélico irlandés, Bizkaia, Irlanda, Edad Moderna, Migración.

Irish language in early modern Biscay: Early modern Lordship of Biscay hosted a small community of Catholic Irish refugees, who found ways of prosperity linked to the Atlantic trade, after fleeing from the protestant repression of Ireland. Both within this group and in other public spaces, Irish language played a relevant role in different socioeconomic domains: decrees of the Consulado de Bilbao, court cases, naturalization of Irish immigrants, Irish tannery activities in Biscay, common sociability areas, or the privacy of the Irish families. Irish language found their place in all these environments as a way of claiming and preserving the identity and cultural attributes of an exiled society.

Keywords: Irish language, Biscay, Ireland, Early modern period, Migration.

\section{Introducción}

A lo largo de los siglos XVII y XVIII el puerto de Bilbao representaba un espacio mercantil en el que no solo se producía un intercambio de 
mercancías, sino también de ideas, personas, culturas, etc. En este ir y venir de lo material y lo inmaterial, fruto de la apertura de la villa vizcaína al mundo atlántico, las lenguas empleadas en estas transacciones y en toda la condición relacional que ello implicaba, se manifestaban como un instrumento fundamental.

Así, el puerto de Bilbao, con los años y derivado de las diferentes coyunturas sociopolíticas de la Edad Moderna, fue configurando su particular universo lingüístico como un elemento dinámico y distintivo, al igual que otros grandes enclaves del comercio europeo (Burke 2004: 118; Boone and Porfyriou 2007: 61; Cabantous 1990). Entre las diversas lenguas que componían este entorno idiomático, el irlandés no era una de las mayoritarias, qué duda cabe, pero tuvo su propio espacio y relevancia sociolingüística dentro de tal ámbito, cuyo análisis constituye el objeto del presente trabajo.

Como premisa inicial, cabe considerar la presencia del gaélico irlandés ${ }^{1}$ en Bizkaia desde dos perspectivas diferenciadas, aunque claramente interrelacionadas: la de las tripulaciones de navíos que arribaban a los puertos del Señorío de Bizkaia, y la de la comunidad conformada por los emigrados irlandeses que se asentaron en la villa de Bilbao y su binterland durante la Edad Moderna. Y en esa línea, desde un plano más instrumental, el papel de la lengua irlandesa en la Bizkaia moderna puede abordarse bajo dos enfoques: el uso que de la misma se hacía públicamente en el puerto de Bilbao, esto es, fuera de la propia comunidad de emigrados irlandeses; y el protagonismo que esta tomaba en el seno de la misma.

En cuanto al primer grupo, el irlandés representaba uno de los muchos idiomas que los múltiples barcos foráneos introducían en el puerto de Bilbao; al igual que el francés, el flamenco, el neerlandés, el portugués, el inglés, etc. Sin embargo, representa un elemento atípico, pues en este caso no se guardaba necesariamente una correlación entre el idioma empleado por los tripulantes y el origen del navío. El país de origen en este caso estaba sufriendo una transformación lingüística muy significativa a lo largo de los dos siglos contemplados en este análisis, y el uso del inglés o el irlandés fue una circunstancia cambiante y dependiente de diversos factores, tales como el año, la ciudad o puerto de origen, la clase social del hablante o, incluso, la situación política de cada momento -en especial, al visitar territorios de la Monarquía Hispánica-. Si ya resulta complejo establecer el uso del gaélico dentro de Irlanda durante los siglos XVII y XVIII -como se verá más adelante-, tanto más dificultoso se antoja evaluar su presencia en Bizkaia o, más particularmente, en Bilbao. No obstante, se dispone de ciertas evidencias que permiten teorizar, con mayor o menor certeza, sobre cómo se manifestaba el idioma irlandés en los puertos vascos. Así pues, dentro de esta primera categorización sociolingüística cabría contemplar aquellos gaélico-parlantes que 
llegaban a Bilbao a través de las redes comerciales atlánticas como integrantes de las tripulaciones de navíos, bien como capitanes o bien como marineros. Esta presencia se caracterizaba a grandes rasgos por una temporalidad corta -esto es, la duración que el proceso de carga y/o descarga mantuviera al mercante atracado en la ría bilbaína-, y una localización cercana al puerto, es decir, la propia villa, y principalmente, las riberas de la ría en Abando, Deusto, Olabeaga, Zorroza, o incluso, aguas abajo, el puerto de Portugalete. En este caso, los espacios sociales que ocupaban eran, fundamentalmente, las tabernas, mesones, hospedajes, posadas y otros establecimientos que daban servicios de alojamiento, comida y bebida a la marinería durante su estancia; así como los comercios y talleres que se precisaran para reabastecer la nave antes de su partida.

Por otro lado, y quizá con mayor relevancia sociológica, se encontraba la comunidad de irlandeses católicos migrados desde Irlanda, que durante casi dos siglos llegó huyendo de las políticas opresivas impuestas por la ocupación y dominación inglesa de la isla (Bilbao Acedos 1999 y 2004). A diferencia del grupo anterior, este colectivo representaba un conjunto de individuos asentados en Bizkaia -en la mayor parte de los casos- durante muchos años, con actividades y ocupaciones estables, y redes socio-profesionales en permanente exposición a la sociedad vizcaína. Estos desplazados irlandeses que fueron asentándose en Bilbao y sus alrededores conformaron, a medida que crecía su número, una comunidad extremadamente cohesionada -incluso con algunas de las características endogámicas propias de los colectivos de inmigrantes- en la cual cabe presuponer que se preservaron muchos de los valores culturales de origen. En general, los estudios relativos a este conjunto de emigrados $-\mathrm{y}$, de modo genérico, los relativos a otras comunidades de irlandeses asentados en tierras hispánicas- se han centrado en diferentes aspectos, tales como las motivaciones para abandonar Irlanda, las estrategias de asimilación en la sociedad de acogida, sus actividades económicas y profesionales, y otra serie de consideraciones políticas, económicas y sociales. Sin embargo, queda por llevar a cabo un estudio en mayor profundidad sobre el grado de implantación que la cultura irlandesa tuvo en estos grupos instalados en la península Ibérica, bien de forma global o bien en cada ciudad particular de establecimiento. En este sentido, el presente trabajo pretende cubrir una pequeña parte de este ambicioso análisis, a través de una aproximación al idioma irlandés en el Señorío de Bizkaia, como rasgo identitario y cultural de primera magnitud. ${ }^{2}$ En cualquier caso, quedarían múltiples atributos culturales por abordar, tales como la literatura -tanto oral como escrita-, el arte, la religiosidad, la música, las festividades, la gastronomía, etc.; si bien muchos de los cuales al ser parte de un patrimonio inmaterial resultan difíciles de seguir a través de las fuentes documentales disponibles en nuestros días. 
Por último, se hace preciso mencionar que el presente trabajo se enmarca en un planteamiento general de investigación llevado a cabo por el autor dirigido a evaluar los vestigios -si los hubiera- del legado cultural irlandés en Bizkaia. Sin embargo, hay que considerar que en términos cuantitativos la comunidad irlandesa de la Bizkaia moderna fue muy reducida, si se compara con la de otros asentamientos de irlandeses de la época, tanto en Europa -St Malo, Burdeos, Bayona, Lisboa, etc.- como en España - principalmente, Cádiz, y en menor medida, La Coruña, Málaga, la corte madrileña, etc.-. Este hecho, junto con la cuestión de que los irlandeses dejaron de establecerse en Bizkaia a finales del siglo XVIII, plantea la hipótesis de que las sucesivas generaciones fueron absorbidas por la sociedad y cultura vizcaínas, y el legado cultural irlandés fue degradándose en la misma medida. A pesar de que hoy en día siguen presentes en Bizkaia algunos de esos apellidos irlandeses, muchos de sus descendientes desconocen ese origen, o lo han descubierto recientemente a través de un creciente interés por la genealogía. En cualquier caso, la mencionada línea de trabajo sigue abierta.

\section{El gaélico en Irlanda}

Para entender la presencia y relevancia que el gaélico tuvo en tierras vizcaínas es imprescindible conocer la situación y evolución del mismo en tierras irlandesas. Sin embargo, la aproximación a la situación lingüística de Irlanda durante los siglos XVII y XVIII no resulta una tarea sencilla, puesto que las fuentes documentales al respecto son prácticamente inexistentes, $y$, en todo caso, la lengua irlandesa se inclinaba hacia la comunicación oral mientras que los registros documentales estuvieron desde años muy tempranos ligados al empleo del inglés (Keegan 2013: 80-1). Sin embargo, a lo largo de las últimas décadas se han llevado a cabo investigaciones de rigurosidad contrastada que han abordado el reto de plasmar la situación geolingüística de la isla a través de distintas aproximaciones metodológicas, las cuales han permitido conformar unos valiosos mapas estimativos del empleo del gaélico, incluso en ciertos estudios, para diferentes momentos históricos (Ravenstein 1879, Ó Cuív 1969, Hindley 1990, Mac Giolla Chríost 2004, O’Néill 2005, Smyth 2006, Doyle 2015).

En 1851 se realizó el primer censo de población en Irlanda que contemplaba el perfil lingüístico de sus habitantes, y así se fueron repitiendo sucesivamente con una periodicidad decenal hasta bien entrado el siglo XX. A partir de estos datos, el geógrafo E. G. Ravenstein llevó a cabo en el siglo XIX el primer análisis estadístico del empleo del irlandés dentro de su estudio general sobre las lenguas célticas de las Islas 
Británicas, donde el citado idioma copaba una parte muy sustancial del trabajo. Fue pionero a la hora de elaborar mapas de convivencia lingüística entre el irlandés y el inglés en Irlanda, con las baronías como unidad geográfica, a partir de los mencionados censos de población de 1851 y 1871. De modo general, se percibe cómo en 1851 las zonas con mayoría de gaélico-parlantes se concentran en la parte occidental de la isla, con la franja central como un área de convivencia entre inglés e irlandés, y una vasta extensión de terreno en la parte central y en la línea oriental del país donde el porcentaje de presencia de este último se reduce agudamente. Veinte años después, en 1871, se aprecia cómo las zonas en las que se hablaba la lengua vernácula se van difuminando y van limitándose a pequeños reductos en la costa oeste. De algún modo, este estudio vino a constatar la regresión y sucesiva desaparición que la lengua céltica había y estaba sufriendo durante ese siglo XIX. Asimismo, la estadística planteada lleva a cabo un exhaustivo desglose numérico por edad, sexo, entornos geográficos, etc. En general, una de las grandes conclusiones que parecen extraerse, más allá del detalle, es que en los años mencionados una gran proporción de la población era bilingüe con claras desviaciones en función del condado (Ravenstein 1879).

A partir de este hito, se puede considerar que la siguiente gran contribución a la definición de la situación del gaélico en la Edad Moderna -o al menos, una de las que más ha trascendido- vino en los años 70 del siglo XX, de la mano del ilustre historiador y filólogo del celtismo Brian O Cuív (1976), quien realizó varios estudios en profundidad sobre el irlandés desde diversos puntos de vista. Desde un enfoque demográfico retomó los datos de los censos previamente descritos y ahondó en los condicionamientos históricos que condujeron la trayectoria lingüística del país, así como otros aspectos más filológicos relacionados con la lengua céltica. Elaboró con la ayuda de la Ordnance Survey of Ireland nuevos mapas más detallados que recogían la distribución idiomática de la isla (Ó Cuív 1969).

Dos décadas más tarde, Reg Hindley (1990) publica, con el elocuente título de The death of the Irish language, un nuevo ensayo donde describe la evolución de la lengua nativa de acuerdo a un discurso histórico-político, y donde lleva a cabo una serie de contribuciones sobre los estudios de Ó Cuív, complementándolos con información más detallada para el norte de Irlanda.

En 2004 y 2005, respectivamente, Diarmait Mac Giolla Chríost y Diarmuid O'Néill publicaron sendos trabajos que recogían las investigaciones previas y llevaban el estudio del reparto geográfico un paso más allá. Centrando el análisis principalmente en el primer caso, el autor propuso por primera vez, a partir de metodologías estimativas, una evolución temporal de la situación etnolingüística irlandesa alrededor de 
1500, alrededor de 1700, a finales del s. XVIII, y a comienzos y finales de los s. XIX y XX. De modo general, resume la situación del gaélico durante la Edad Moderna, a partir de la síntesis de un importante número de estudios al respecto, del modo descrito a continuación. Considera que, a finales del siglo XVII, tras el período de conquista y colonización inglesa, se podían distinguir dos áreas de penetración lingüística sobre el irlandés. La primera se ubicaba en la parte sur y sureste de la isla, abarcando una extensión entre el Pale y Wexford al este hasta Limerick en el oeste, y el Bog of Allen y las Slieve Bloom Mountains en el norte hasta el río Nore y las Slievedargh Hills en el sur. Fuera de este ámbito geográfico, se podían encontrar comunidades significativas de angloparlantes, particularmente en centros urbanos, tales como Limerick, Cork, Waterford, Youghal y Kinsale. En las zonas limítrofes con esta región se podía encontrar un área de transición donde el irlandés permanecía como el idioma cotidiano de la mayoría de la población. La segunda zona de penetración del inglés se localizaba en la provincia de Ulster con un patrón geográfico y etnolingüístico mucho más complejo. Por añadido, en una segunda fase, se analiza la evolución del irlandés durante el siglo XVIII, la cual se justifica vinculándola a la construcción del estado-nación de Reino Unido y el papel que Irlanda tuvo en tal proceso. En definitiva, nuevamente, queda plasmada en los mapas etnolingüísticos presentados la regresión de la lengua céltica. Mientras que alrededor de 1700 el espacio predominante del irlandés en la isla ocupa prácticamente todo el oeste y gran parte de la franja central, limitándose puntualmente la predominancia anglófona a las zonas del Pale y una pequeña parte del condado de Wexford; en 1800 , la situación se ve revertida y la mayor parte de la mitad este del país es feudo del inglés, pasando a ser el resto de la isla un espacio bilingüe anglo-irlandés (Mac Giolla Chríost 2004).

También en esos mismos años, y completando la información previa, aparecen las valiosas y novedosas aproximaciones metodológicas y contribuciones del profesor William J. Smyth (2006), quien combina tanto la dimensión geográfica como la socio-étnica de la expansión del inglés. Amplía las fuentes empleadas al trabajar sobre la información de las poll-tax de 1660, las deposiciones de 1641, y las evidencias de apellidos ingleses y escoceses en tales fechas. En cuanto a la presencia geográfica del inglés, en torno a finales del siglo XVII se correlaciona en gran medi$\mathrm{da}$ con las conclusiones obtenidas y representadas por Mac Giolla Chríost. En su cálculo de las comunidades idiomáticas, Smyth estima que aproximadamente un tercio del país podía hablar inglés, aunque muchos de ellos también hablaban irlandés. Y arroja un cálculo que apunta a que el $80 \%$ del país sería gaélico-parlante, muchos de los cuales serían además bilingües. 
El repaso bibliográfico concluye con la gran compilación realizada por Aidan Doyle (2015) sobre la historia de la lengua irlandesa, donde se integran todas las aportaciones científicas realizadas hasta la fecha sobre la temática tratada en un volumen que sintetiza los contenidos expuestos previamente, además de otros muchos aspectos. A buen seguro, representa una de las obras de referencia para el estudio de la lengua irlandesa en los próximos años, incidiendo de modo muy especial en la representación geográfica de la realidad idiomática, en muchos casos a partir de mapas previos.

A grandes rasgos, a partir de todos los estudios presentados, la situación y evolución del irlandés en la isla podría resumirse con las siguientes ideas genéricas. A comienzos del siglo XVII la mayor parte de la población irlandesa hablaba gaélico (Coolahan 2010: 3). Sin embargo, según avanzaba el siglo, en la costa este de la isla, y principalmente, en los entornos urbanos, comenzó a aflorar un bilingüismo derivado de los centros de poder que suponía la presencia de cargos gubernamentales anglófonos y que dictaban las leyes en un marco de superioridad lingüística para el inglés. Así, a comienzos del siglo XVIII, casi la mitad este del territorio irlandés hablaba inglés, bien es cierto que en muchos casos en un marco bilingüe. No obstante, es importante hacer notar que este bilingüismo estaba caracterizado en la mayor parte de los casos por una marcada diglosia, esto es, dos lenguas usadas por una misma comunidad, cada una de ellas para diferentes situaciones económicas y sociales; y marcada también por un bilingüismo transicional-generacional, es decir, abuelos monóglotas de habla irlandesa con hijos bilingües y nietos monóglotas de habla inglesa (O’Gráda 2013). Así, a lo largo del siglo XVIII se dio un fenómeno de sustitución lingüística, geográficamente de este a oeste, que para comienzos del XIX había dejado únicamente unos reductos del irlandés en la costa occidental de la isla (De Fréine 1978).

\section{La presencia del gaélico en Bizkaia}

El puerto de Bilbao fue durante los siglos XVII y XVIII un punto de confluencia comercial de los más destacados del Atlántico, derivado de sus extensas y buenas redes mercantiles con otros puertos de la Europa atlántica y Norteamérica. Ese contexto socioeconómico propició tanto la entrada de navíos que nutrieran las mencionadas relaciones mercantiles como un prometedor entorno de progreso que alentaba a asentarse en la región a diferentes grupos sociales. En ambos colectivos la presencia irlandesa fue notable, y como consecuencia, el gaélico pasó a formar parte del entramado idiomático del territorio. 


\subsection{Navíos y tripulaciones}

Los libros de averías custodiados en los fondos del Archivo Histórico del Consulado de Bilbao son una de las mejores pruebas históricas que detallan la entrada y salida de navíos, incluyendo en su información, en muchos casos, los puertos de origen o destino de los mismos, sus propietarios, sus capitanes y maestres, e incluso la nacionalidad de sus tripulaciones; principalmente desde el siglo XVI al XVIII. A partir de los registros brutos, o de la infatigable labor de extracción y análisis de los mismos llevado a cabo por unos pocos estudiosos de la materia (Guiard Larrauri 1972; Zabala 1994), puede demostrarse cómo la villa bilbaína se conectaba de forma común y fluida con los puertos irlandeses, asentando no menos de 50 navíos anuales que conectaban ambos territorios. Estos datos pueden complementarse con la presencia de un alto número de capitanes y marineros irlandeses que operaban tanto en los propios buques de nacionalidad irlandesa como en los mercantes ingleses, práctica muy habitual en el caso de la marinería. Todo en su conjunto deja plasmado cómo el comercio bilbaíno atrajo un significativo número de irlandeses que, con carácter más bien temporal, recalaron en los muelles vizcaínos, en muchos casos de forma recurrente integrando parte de barcos que mantenían rutas estables en los negocios atlánticos.

En este sentido, la presencia irlandesa ligada a los barcos que integraban el comercio bilbaíno no queda únicamente plasmada en el origen de los mismos, sino que el propio Consulado de Bilbao reconocía esta circunstancia y consideraba de relevancia e importancia para la villa tanto la comunidad irlandesa como su lengua, tal como se describe a continuación. Las Ordenanzas del Consulado de 1737 establecían y regulaban el número y la labor de los corredores de navíos presentes en el puerto de Bilbao, quienes debían intermediar en las negociaciones, contratos y ajustes entre los agentes extranjeros y las casas de comercio locales facilitando en la mayor parte de los casos servicios de traducción. Así, en la designación de los mismos, llevada a cabo por el propio Consulado, este reconocía las capacidades lingüísticas de sus corredores en la lengua gaélica como un hecho relevante; y de tal modo se apuntaba en las competencias de los cuatro corredores. En 1738, un año después de la entrada en vigor de las mencionadas Ordenanzas, el Corregimiento vizcaíno llevaba a cabo las diligencias oportunas para el control y prevención de la peste generada en varios países europeos, para lo cual comunicaba a sus cuatro corredores de navío -Phelipe Duberon, Miguel Archer, Juan de St Aulary y Juan Bauptista Nougaro- las medidas a tomar y comunicar en relación a los barcos que arribaran al puerto. En tal circular se describía al corredor irlandés como: “(...) Miguel Archer corredor de nabios 
y interprete de las lenguas franzesa, ynglesa y yrlandesa (...)" ${ }^{3}$ No sería esta la única vez en la que Archer prestaría servicios como traductor de gaélico para el Consulado de Bilbao; por ejemplo, en 1734, se ocupó de los servicios de interpretación demandados por el Consulado en la declaración de cuatro marineros irlandeses "(...) como yntelixente en ella [lengua castellana] y en la irlandesa (...)”. ${ }^{4}$

Al hilo de este apunte resulta sintomático que pocos años más tarde, tras el fallecimiento del irlandés Terencio MacMahon en 1766, quien había ejercido también durante varios años como corredor de navíos en Bilbao, los candidatos a su sucesión presentaron carta de solicitud para la plaza vacante acreditando sus capacidades lingüísticas, y en ninguna de las tres solicitudes presentadas se denota el dominio del irlandés. Esta afirmación es más relevante aún si se considera que uno de los aspirantes, y quien finalmente acabaría ocupando el cargo, fue Patricio MacMahon, hermano de Terencio. En su carta de presentación indicaba “(...) hallarse asistido con la apreciable abilidad de saviduria en las lenguas española, inglesa, francesa, portuguesa y latina (...)". ${ }^{5}$ Ambos hermanos procedían del condado de Cork, región que integraba uno de los reductos más sólidos del gaélico en Irlanda en el siglo XVIII, lo que hace pensar con casi total certeza que hablaran la lengua vernácula, y que la razón de que no se apuntara entre sus capacidades idiomáticas respondiera a que el uso y la relevancia del irlandés en Bizkaia había ido decayendo a lo largo del siglo, cuestión que se corresponde con la realidad lingüística sufrida en la isla.

Además de los servicios requeridos por el Consulado -en el caso de los corredores de navío- o por el consistorio -en el caso de las probanzas de hidalguías, como se verá más adelante-, otras instituciones vizcaínas precisaron de estos servicios de traducción en respuesta a procesos judiciales que reflejaban la cotidianeidad de la presencia marinera irlandesa en la vida pública vizcaína. Así, en 1642 Raimundo Esqueret, mercader irlandés residente en Bilbao y dueño del navío Margarita, salía en defensa de un marinero irlandés de nombre Tadeo contra Juan Martínez de Sarria, por cortar un cable de dicha embarcación y lesionar al citado marinero. ${ }^{6}$ En 1694, el irlandés Bernardo Gorcio denunciaba el hurto de trece doblones perpetrado por el marinero inglés Felipe Born. ${ }^{7}$ En 1710 el Corregidor vizcaíno, actuando de oficio, denuncia a los hermanos Zulueta por lesiones ocasionadas a un marinero irlandés llamado Thomas Torbott. ${ }^{8}$ Nuevamente actúa de oficio el Corregidor en 1716, esta vez contra Diego Guiyens, marinero irlandés, denunciando los golpes y amenazas lanzados contra Juan de Escalza y contra el también irlandés Thomas Moriarte, mientras cuidaban el navío San Juan..$^{9}$ De nuevo de oficio, pero esta vez el alcalde de Bilbao, se denuncia en 1725 al capitán John Rocastle, dublinés, por amenazas al marinero irlandés 
Diego Arnes. ${ }^{10}$ En 1732, se acusa al capitán inglés Thomas Dowen de agredir al marinero irlandés Thomas Lew. ${ }^{11}$ En 1738 se denunciaba a un marinero irlandés de nombre Eduardo y a Margarita Cranmester, viuda residente en Bilbao, por escándalo público y amancebamiento. ${ }^{12}$ Dos años más tarde, Eduardo Bream, marinero irlandés, inicia causa criminal contra el capitán inglés Guillermo Piamar por injurias y malos tratos. ${ }^{13}$ Y, como los presentados, pueden encontrarse otras tantas causas judiciales que involucran a marinería y capitanes irlandeses; respondiendo, en general, a agresiones por parte de los capitanes a sus tripulaciones -siendo el de nación irlandesa tanto el capitán como el marinero-, hurtos y robos; o casos de escándalo público, relaciones ilícitas e, incluso, estupro. En cuanto a esta última categoría, no solo aparecen marineros irlandeses, sino que también existen sucesos en los que son irlandesas las acusadas de escándalo público, tales como Margarita O’Connor o Ana Cabanax. ${ }^{14}$ Aunque no queda claro el contexto de estos incidentes, son varias las irlandesas que se conocen avecindadas en Deusto y Olabeaga -como taberneras o regentando pensiones-, esto es, en el entorno portuario nuevamente, lo que sugiere que estos sucesos estuvieran conectados con las tripulaciones de los navíos atracados en la ría del Nervión.

Estos episodios de conflictividad ponen de manifiesto una realidad que era común a toda la sociedad vizcaína, y que se producía con particular intensidad en el entorno portuario de la ría de Olabeaga y Deusto (Grande 2015: 220, 222; Bernal 2011: 489). Más allá de los hechos en sí, no deja de ser un indicador de cómo los marineros irlandeses, y por extensión su lengua, formaban parte de los espacios de sociabilidad cotidianos de los vizcaínos, muy especialmente en el ámbito de las tabernas, que representaban el principal entorno de esparcimiento y ocio, más acentuado aún para el caso de las tripulaciones que atracaban en los muelles bilbaínos. En estos ámbitos sociales, a pesar de los altercados que se pudieran producir en ciertos momentos con carácter circunstancial, se aprovechaba para distraerse de la rutina laboral y, en gran medida, para compartir información (Bernal 2003: 410), en la lengua que mejor se ajustara a los interlocutores.

Este último aspecto resultaba particularmente relevante puesto que permitía y propiciaba la interacción entre las dos comunidades irlandesas que conformaban parte del tejido social vizcaíno, a saber, las tripulaciones de nacionalidad irlandesa que visitaban el puerto bilbaíno y la comunidad irlandesa que habitaba permanentemente en el Señorío de Bizkaia. Que los miembros de ambos grupos frecuentaban las tabernas vizcaínas es un hecho constatado en los registros de archivo; ${ }^{15} \mathrm{y}$, por lo tanto, cabe pensar que interaccionaran tanto por los propios intereses comerciales que pudieran surgir como por la necesidad de intercambiar información en cuanto a la situación socio-política de ambos países y en cuanto a lo personal para tener noticias de los familiares y seres queri- 
dos que vivieran a un lado y otro del mar Cantábrico. Sin duda, en un plano más profesional, durante las transacciones, acuerdos, cargas, descargas y otras operaciones que se produjeran en el entorno portuario, los flujos de información serían igualmente bien acogidos por ambas partes. En todas estas conversaciones entre compatriotas cabe presuponer que la lengua irlandesa fuera la protagonista en parte de los casos, aunque ciertamente resulta difícil de cuantificar y de discriminar en qué medida sería esta la vía de comunicación o lo sería el inglés, atendiendo a la convivencia de ambas lenguas descrita en la sección anterior.

\subsection{La comunidad irlandesa de Bizkaia}

Resulta extremadamente complejo aportar datos detallados sobre el número de gaélico-parlantes en el Señorío de Bizkaia, principalmente por dos motivos. En primer lugar, durante los siglos XVII y XVIII no se dispone de censos de población lingüísticos y, mucho menos, para una lengua tan minoritaria como sería el irlandés. ${ }^{16}$ En segundo lugar, el irlandés fue una lengua, por tradición cultural y por la propia evolución política de Irlanda, con vocación puramente oral. Sin embargo, se dispone de datos suficientes para llevar a cabo unas estimaciones bastante precisas acerca del uso del gaélico en la comunidad irlandesa de la Bizkaia moderna. ${ }^{17}$

Como condición genérica de partida, es asumible que la totalidad -o, al menos, la práctica totalidad- de los irlandeses asentados en Bizkaia formaran parte de los contingentes de católicos que fueron desplazados hacia el continente a resultas de la ocupación inglesa y las consiguientes políticas discriminatorias impuestas hacia la población católica. Esta asunción implica que dicha población pertenecía a los grupos étnicos de Old Irish o Gaelic Irish -población originaria de Irlanda y de habla irlandesa-, y los Old English o Anglo Normans -descendientes de los normandos que invadieron Irlanda durante los siglos XII-XIII y que, con el paso del tiempo, asimilaron gran parte de las costumbres y cultura de los habitantes nativos, los cuales se regían por el orden gaélico (Smyth 2006: 441)-, herederos todos ellos de la tradición cultural irlandesa y, por extensión, de su idioma. ${ }^{18}$ De este modo, se considerará como premisa inicial que todos los migrados irlandeses hablaban potencialmente el irlandés. Sin embargo, ya se ha expuesto previamente cómo en la propia isla no toda la población católica hablaba gaélico, sino que la distribución etnolingüística no era uniforme ni estable en el tiempo. Puesto que la ordenación geográfica del irlandés dependía claramente de las regiones o condados donde se habitara, resulta preciso disponer de una clasificación sobre el origen de los irlandeses vizcaínos. Esta información ha podido extraerse a partir de los 
registros sacramentales de los irlandeses de Bizkaia, donde en un gran número de casos se establece su lugar de procedencia, y a partir de ello, se ha podido elaborar un reparto por condados de origen (Cagigal 2018). ${ }^{19}$ Contrastando los mapas de distribución etnolingüística del irlandés para los siglos XVII y XVIII, y los mapas de procedencia de los irlandeses asentados en Bizkaia, pueden extraerse importantes conclusiones sobre el uso potencial del gaélico en tierras vizcaínas. ${ }^{20}$

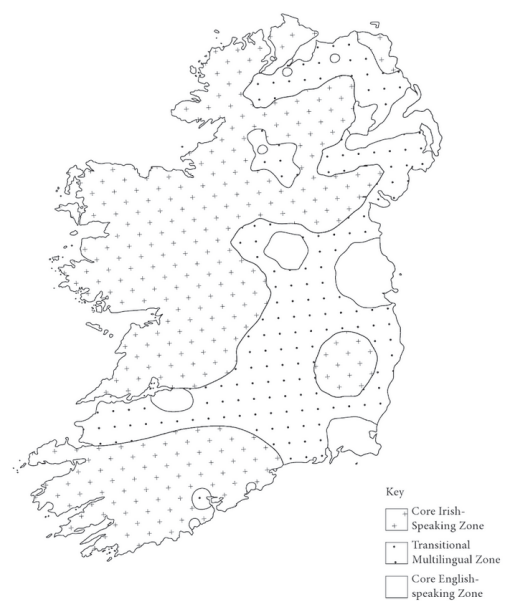

(a) Comunidades lingüísticas en Irlanda c.1700 (Mac Giolla Chriost 2005)

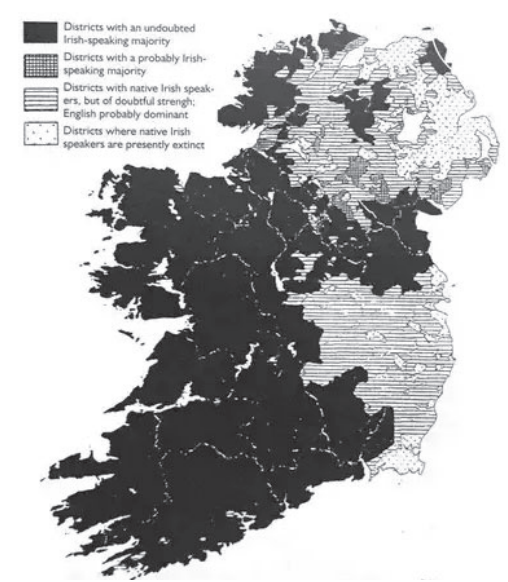

(c) Comunidades lingüísticas en Irlanda c. 1851 (Cuív 1969)

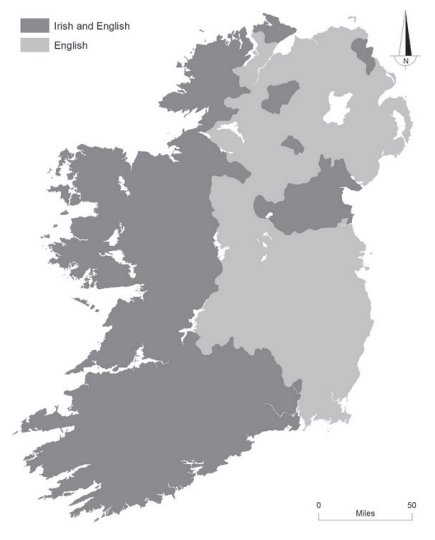

(b) Comunidades lingüísticas en Irlanda c.1800 (Doyle, 2015, based on Mac Giolla Chriost, 2005 y Fitzgerald 1984)

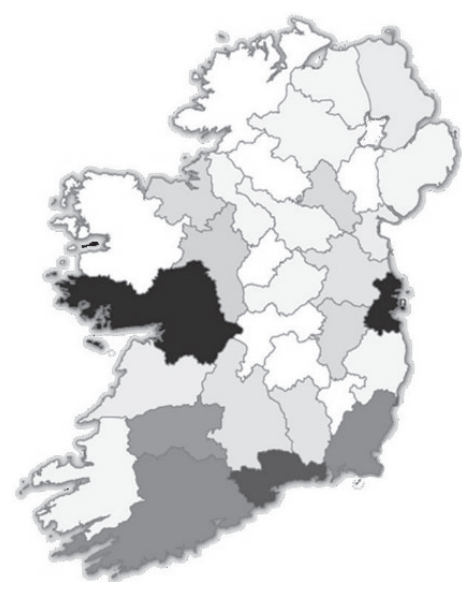

(d) Distribución geográfica por condados de origen de los irlandeses de Bizkaia (Cagigal 2018)

Figura 1. Distribución etnolingüística en Irlanda en los años 1700 (a), 1800 (b) y 1851 (c), y origen de los emigrantes irlandeses de Bizkaia por condado (d).

Lengua y migración / Language and Migration 12:1 (2020), 149-173

Edición impresa: ISSN 1889-5425. Edición en línea: ISSN 2660-7166. (C) Universidad de Alcalá 
La comparativa entre ambos grupos de datos indica que alrededor de 1700 el $75 \%$ de los irlandeses asentados en Bizkaia hablarían irlandés, mientras que en 1800 el porcentaje oscila entre el 56 y el $70 \%$ en función de la fuente empleada (Hindley 1990; Mac Giolla Chiost 2004). Considerando que, según lo expuesto en el apartado anterior, la evolución del habla irlandesa fue decayendo a lo largo del siglo XVII y, principalmente, a partir de la segunda mitad del siglo XVIII, es de suponer que los porcentajes indicados fueran superiores al $75 \%$ antes de 1700 y que oscilaran entre el 56 y el $75 \%$ durante el siglo XVIII. Al margen de la distribución geográfica por condados, estas estimaciones deberían incluir consideraciones adicionales, tales como el estrato social al que perteneciera y el entorno del que procedía cada individuo, atendiendo a que el gaélico era mayoritariamente hablado por las clases más bajas de la sociedad, y a que los ámbitos rurales preservaban en mayor grado la lengua irlandesa frente a los entornos urbanos. Esta observación implica que el decaimiento general de usuarios del irlandés sufrido durante el siglo XVIII podría haber sido compensado en Bizkaia por los dos condicionantes indicados, dado que durante la primera mitad del siglo se produjo la llegada de un número considerable de artesanos que dotaban de mano de obra cualificada a los talleres de curtido de pieles fundados e impulsados por irlandeses en Bizkaia, los cuales pertenecían en gran parte a la clase social de artesanos y eran en su práctica totalidad oriundos de enclaves rurales (Bilbao Acedos 1999). Así pues, de acuerdo con esta comparación, puede sostenerse que una gran mayoría de los irlandeses establecidos en tierras vascas durante la Edad Moderna hablaban gaélico. Por añadido, la nueva realidad y la libertad política que propiciaba su asentamiento en Bizkaia hace presuponer que hicieran uso de su lengua en las relaciones intracomunitarias, a modo de signo de identidad etno-cultural.

Otro medio que puede emplearse para llevar a cabo una valoración de la presencia del gaélico en el Señorío de Bizkaia tiene que ver con el grupo étnico al que pertenecían los emigrados. A pesar de que anteriormente ya se ha justificado de forma genérica el colectivo social al que, a priori, pertenecería tal grupo de inmigrantes, puede llevarse a cabo un análisis de las familias étnicas presentes en Bizkaia en base a los apellidos de los mismos, los cuales se clasifican fácilmente etno-históricamente. Según tal información, el $52 \%$ de los nombres recopilados en estudios previos (Cagigal 2018) responden a apellidos catalogados como Old Irish o Gaelic Irish, el 18\% de los mismos a apellidos Old English o Anglo Normans, mientras que el $30 \%$ restante responde a un origen New English, u otros apellidos cuya categorización resulta incierta o, simplemente, se desconoce. Puede apreciarse cómo esta estimación presenta una clara correlación con el análisis por condados presentado previamente, y que viene a ratificar el alto grado de individuos procedentes 
de la cultura -y, por lo tanto, de la lengua- gaélica, tanto los propios Old Irish como los Old English, descendientes de las invasiones normandas del siglo XIII, quienes con el tiempo adoptaron, en gran medida, las costumbres y la cultura del orden gaélico.

Más allá de aproximaciones estimativas, hay ciertos fondos de archivo que atestiguan documentalmente esta realidad, y que, además, permiten apuntar a una cierta correlación con la evolución del irlandés en la isla. Estas evidencias tienen que ver con las pruebas de hidalguía y probanzas de sangre, que la ley foral del Señorío de Bizkaia exigía para la naturalización y avecindamiento en sus territorios. Tales trámites consistían básicamente en acreditar la genealogía del aspirante y demostrar así su limpieza de sangre, esto es, la inexistencia de antepasados que pertenecieran a otras religiones. Para ello, era preciso, además de las pruebas documentales que se pudieran presentar, la declaración de, al menos, tres testigos que certificaran la genealogía presentada, lo cual para el caso de aspirantes extranjeros implicaba testigos de su mismo país que hubiesen conocido a padres y abuelos. De este modo, en los primeros procesos de avecindamiento defendidos en Bizkaia por irlandeses durante el siglo XVII, para aquellos testigos que no eran religiosos -considerando que los religiosos, por lo general, eran más instruidos en lenguas-, surge recurrentemente la necesidad de traductores que pudieran transmitir a las instituciones forales sus testimonios. Así, en 1663 en la documentación de la probanza de hidalguía de Thomas Bodquin se detalla: "tema que presentar algunos $\mathrm{tt}^{\text {tos }}$ [testigos] que no ablan bien la lengua castellana porque heran irlandeses, pedía y suplicava a sus mrds [mercedes] se sirvan de nombrar ynterprete que entienda la dha lengua". Para tales servicios se nombraría traductor a Fray Antonio del Rosario, dominico irlandés residente en Bilbao. ${ }^{21}$ En 1661, en el proceso de Guillermo Kelly, se declara lo siguiente: "se han examinado diferentes testigos pero para hacerla en cumplida forma ttengo necessidad de presentar algunos que no saven la lengua castellana y los que assi tengo que presentar y no saven ottra que la irlandesa la qual ttanpoco la entiende el dicho sindico ni el presentte escribano". En este caso ejercería de intérprete el clérigo irlandés Francisco de Joseo. ${ }^{22}$ En similares términos demandaba los servicios de traducción Miguel Morgan en 1690 para la defensa de su hidalguía: "se nombre interprete para los testigos que no supieren mas de la lengua inglesa o irlandesa". El encargado de la traducción en este caso sería el padre dominico Juan Reily. ${ }^{23}$ Y, análogamente, sucedería con los procesos de naturalización de David Englando (1652-3), Miguel Hor (1674), Pedro Madan (1673) y otros irlandeses. ${ }^{24}$ En general, esta era una circunstancia que se dio repetidamente en las declaraciones de testigos irlandeses durante el siglo XVII, siempre que estos no fueran religiosos, incluso en ciertos casos en los que el deponente llevaba viviendo varios años en dominios de la Monarquía Hispánica. Por el contrario, una vez entrado el siguiente siglo 
resulta inusual encontrar demandas de traducción en los procesos de probanzas de genealogía, lo cual respondería a dos razones principales. En primer lugar, los testigos presentados eran, en su práctica totalidad, individuos que llevaban residiendo en Bizkaia varios años, con lo que hablaban, con mayor o menor fluidez, la lengua castellana. Y, en segundo lugar, aquellos que hubieran llegado de Irlanda más recientemente hablarían inglés con mayor probabilidad, lo cual facilitaba la tramitación con los cuerpos administrativos del Señorío, bien porque los síndicos, escribanos y otros cargos conocieran tal idioma, o bien porque la traducción del inglés fuera más accesible. Este último hecho presenta una coherencia con la evolución idiomática de la Irlanda moderna presentada en los apartados previos.

Además de los análisis genéricos presentados merece la pena ahondar, desde la perspectiva social del uso y presencia del irlandés, en dos grupos sociales específicos que integraban la comunidad irlandesa de Bizkaia: el Hospicio de las Calzadas de Begoña, fundado por un pequeño grupo de religiosos irlandeses pertenecientes a la Orden de Predicadores; y las tenerías o curtidurías dirigidas por irlandeses que funcionaron en Bizkaia durante el siglo XVIII.

Desde mediados del siglo XVII y hasta finales del siglo XVIII habitaron en unas casas de las Calzadas de Begoña de Bilbao un pequeño grupo de dominicos irlandeses, que vivían en condiciones de extrema austeridad, hasta el punto de que en 1697 el superior de la congregación solicitó a la Diputación una ayuda económica, que se materializó en una limosna de 500 reales anuales (Cifuentes Pazos 2003; Labayru 1971). En contraprestación, estos religiosos se ofrecieron a actuar como traductores para las autoridades vizcaínas en aquellos casos en los que sus servicios fueran necesarios, figurando habitualmente en la documentación de archivo relativa a pleitos judiciales, procesos de naturalización, protestas de mar, etc. Además de ello, estos frailes se dedicaron particularmente a asistir marineros extranjeros, principalmente ingleses e irlandeses, que acogían en el hospicio fundado en su residencia. En este marco, cabe presuponer que el Hospicio representara un pequeño refugio de la lengua irlandesa, donde era empleada por los propios religiosos para comunicarse entre ellos -fuera de los espacios donde la regla monástica dictara el uso del latín-, así como con algunos de los marineros acogidos en el albergue. Por añadido, muchos de ellos atendían, a su vez, las necesidades espirituales de la comunidad irlandesa que habitaba en el entorno de Bilbao, para lo cual su lengua materna resultaría ser la adoptada comúnmente.

Fueron otros muchos los religiosos irlandeses que residieron en los conventos bilbaínos de San Agustín, San Francisco y Santo Domingo, algunos con carácter permanente y otros con mayor o menor temporalidad, en su salida hacia el norte en la denominada Misión de Irlanda. Sin embargo, este colectivo de irlandeses se integraba en las congregaciones 
vizcaínas lo que induce a considerar que su interlocución en irlandés se limitara a las relaciones extra-conventuales o con los compatriotas que pudieran encontrarse en ese momento en privado. A pesar de que no hay constancia de que tal polémica afectara a las comunidades de religiosos irlandeses asentadas en Bizkaia, merece la pena señalar que durante el siglo XVII la cuestión de la lengua fue un elemento de fuertes discrepancias entre varias órdenes de religiosos irlandeses durante su expatriación. Mientras que los jesuitas abogaban por la formación de sus estudiantes en latín, los franciscanos defendían la necesidad de mantener también el irlandés como idioma de aprendizaje con el fin último de que aquellos religiosos que retornaran a la isla en la denominada Misión de Irlanda pudieran atender las necesidades de sus feligreses en la lengua vernácula (O’Connor 2017: 100).

Otra de las razones que llevan a pensar que la comunidad irlandesa de Bizkaia preservara notablemente el uso del irlandés tiene que ver con las prácticas sociales en las que se movían, las cuales les retratan como un grupo fuertemente cohesionado, hasta el punto de poder entreverse como altamente endogámicas. Esta realidad se manifestaba particularmente en las curtidurías dirigidas por irlandeses. Tales talleres artesanales fueron en su mayor parte fundados por irlandeses, e incluso, en aquellos casos en los que los propietarios o inversores de tales negocios eran vizcaínos, estos delegaban la dirección de los mismos en maestros irlandeses. Además, ocurría a menudo que los maestros buscaban y facilitaban la contratación de oficiales y aprendices procedentes, asimismo, de Irlanda para garantizar las prácticas de curtido a la irlandesa, las cuales eran muy apreciadas en la península Ibérica. Y por añadido, como era práctica habitual en los gremios artesanales del Antiguo Régimen, los oficiales acababan contrayendo matrimonio con las hijas de sus maestros, e incluso, en ciertos casos, heredando el negocio, fenómeno que se produjo con asiduidad entre los curtidores irlandeses del Señorío de Bizkaia (Bilbao Acedos 1999). Otro elemento aglutinador desde el punto de vista profesional eran las actividades asociativas y/o mancomunadas entre diferentes maestros curtidores irlandeses para la compraventa de sus manufacturas y para el abastecimiento de materias primas, relaciones que generalmente trascendían lo profesional hacia ámbitos de sociabilidad mucho más personales. Puede percibirse cómo la tenería conformaba un reducto de lo irlandés, esto es, un ámbito social tan demarcado y restringido, que incitara a reproducir los usos y costumbres relacionales propios de la isla, entre los cuales, qué duda cabe, la lengua jugaría un papel fundamental y encontraría un espacio natural en el que manifestarse.

Además de los ambientes sociales descritos, la familia fue, sin lugar a duda, el ámbito en el que la lengua irlandesa se desarrollaría con mayor 
intensidad, principalmente por dos razones. La primera es que representaba un espacio personal en el que el individuo se podía expresar sin ningún tipo de coerción, y en los matrimonios donde ambos hablaban irlandés se darían las circunstancias naturales para que la comunicación, al menos en privado, fuera en este idioma. ${ }^{25}$ En segundo lugar, las comunidades de inmigrantes expulsadas de su tierra exhiben una alta propensión a mantener y preservar los rasgos culturales que les definen como grupo en el exilio, al punto de trasladar a sus descendientes estos atributos, de tal modo que pervivan aun desplazados de su tierra materna. Y en este contexto, fueron numerosos los hijos de irlandeses que aprendieron la lengua nativa de sus padres a pesar de haber nacido en tierras vizcaínas, dotándoles así de un rasgo identitario que la expulsión les había privado. ${ }^{26}$

De forma global, y considerando que el idioma es un rasgo preponderante de la cultura de un pueblo, hay ciertas pruebas que demuestran cómo la comunidad irlandesa de Bizkaia trató de mantener sus atributos identitarios, además de la propia lengua. Así lo demuestra, por ejemplo, la iniciativa de Valentín Morgan para la fundación de la capilla de San Patricio, patrón de Irlanda, en el convento de San Agustín de Bilbao, donde fueron sepultados un gran número de estos individuos.

\section{El multilingüismo de los irlandeses en Bizkaia: el caso de Guillermo Magra}

\subsection{Consideraciones generales}

Como se ha descrito anteriormente, en gran parte de las ocasiones, los emigrados irlandeses que recalaron en Bizkaia -sin entrar en consideraciones sobre la educación de la que hubieran podido gozar- eran capaces de hablar tanto inglés como irlandés. Tras pocos años en España, hablarían e, incluso, dominarían el castellano. Y no era extraño que muchos de ellos hubiesen terminado en Bizkaia tras un paso por Francia, bien por formar parte de los ejércitos del país vecino, bien por participar en el activo comercio francés, o bien por haber formado parte de la corte jacobita en el exilio, lo que sumaría el francés a sus cualidades comunicativas. Por añadido, muchos de estos irlandeses participaban del comercio atlántico, tanto como mercaderes como en los navíos mercantes -esto es, como capitanes o marineros-, lo que añadiría fácilmente otras lenguas, como el flamenco, el holandés o el portugués. De este modo, aunque no de forma generalizada, este colectivo estaría caracterizado por un multilingüismo más o menos acusado en cada caso. 
En cualquier caso, tras todo lo expuesto, no deja de resultar llamativo que en muchas ocasiones los irlandeses asentados en Bizkaia emplearan para determinadas comunicaciones y documentos la lengua inglesa. Sin embargo, muy a su pesar, la sociedad irlandesa de los siglos XVII y XVIII se caracterizaba en gran medida por el bilingüismo, con todas las matizaciones que precisa tal afirmación. Y es que la realidad sociocultural de la época conformó un mapa lingüístico donde el entorno bilingüe era mayoritario, tal como se ha descrito previamente.

Esta situación explicaría por qué Edmundo Shee se carteaba en inglés con su hijo, residente en Cádiz, o por qué muchos comerciantes irlandeses que habitaron en Bilbao decidieron dejar sus últimas voluntades escritas en inglés. Esto podría responder a varias cuestiones: a la realidad idiomática descrita, a un bilingüismo puramente funcional en el que el irlandés ocupaba la comunicación oral mientras que el inglés se empleaba para los documentos escritos, o a que simplemente se hubiera llegado a asimilar el inglés como parte de sus señas de identidad. A buen seguro, cada caso tendría su propia explicación, y en muchas ocasiones se solaparían ciertas dosis de todas estas hipótesis.

De cualquier manera, parece irrebatible argumentar que el mencionado bilingüismo posicionó a los irlandeses de todas las condiciones en una situación social ciertamente ambigua. Ambigua porque eran extranjeros, pero católicos, y particularmente protegidos y considerados por el rey español, lo que les confería una serie de privilegios de los que no gozaban otras nacionalidades (Recio Morales 2002: 316). Sin embargo, hablaban inglés y tenían fluidas relaciones comerciales con los puertos ingleses. Esta circunstancia los llevó a que en determinados momentos fueran rechazados por la sociedad vizcaína, tanto a nivel institucional como en los ámbitos cotidianos. En todo caso, parece claro que su dominio del inglés les proporcionaba una posición privilegiada en las transacciones y contratos comerciales, en la red de contactos y factores comerciales que les representaran en otros puertos, en el propio negocio del corso o el contrabando, y, en general, a la hora de desplegar políticas y estrategias mercantiles del tipo que fueran. Pero no solo en el comercio se materializó esta posición ventajosa, sino que a niveles más modestos también el inglés fue una llave de acceso a otro tipo de negocios. Por ejemplo, se tiene constancia de que varias irlandesas regentaron tabernas, mesones y fondas en el entorno de la ría del Nervión. Y es que aquellos marineros que recalaban en los muelles vizcaínos a buen seguro antepondrían aquellos establecimientos que estuvieran dirigidos por angloparlantes, de modo que su estancia resultara mucho más cómoda en lo comunicativo. Esta ambivalencia socio-lingüística queda extremadamente manifiesta en el caso de Valentín Morgan, oriundo de la ciudad de Waterford, quien tras haber huido de la ocupación inglesa 
de Irlanda, acabaría ejerciendo entre 1661 y 1685 como el primer cónsul inglés de la ciudad de San Sebastián (Santoyo 2008: 53).

No obstante, en un siglo, el XVIII, en el que las relaciones entre la Monarquía Hispánica y la Corona inglesa fueron cambiantes y, en la mayor parte de los momentos, de confrontación bélica, la dualidad lingüística no siempre resultaba algo positivo o provechoso para los irlandeses, sino que por el contrario los llevó a situaciones de malentendidos o injusticias. Sin entrar en valoraciones personales que de los irlandeses podía hacer un determinado individuo vizcaíno, fruto de alguna trifulca o contienda personal, donde el hecho de la nacionalidad podía ser el menor de los agravantes, a nivel institucional sufrieron los agravios de las instancias de poder del Señorío. Por ejemplo, en 1741 la administración vizcaína emitía un listado de comerciantes de Bilbao a los cuales se debía encarcelar y requisar sus bienes, libros y papeles, bajo la acusación de contrabando con los ingleses. Entre ellos figuraban los irlandeses Miguel Archer y Carlos Walcott, también es cierto que junto con otros importantes comerciantes de la villa, tanto locales como extranjeros. ${ }^{27}$

\subsection{El multilingüismo de Guillermo Magra}

Guillermo Magra, ${ }^{28}$ a pesar de formar parte del contingente de curtidores que progresaron en tierras vizcaínas tras su huida de Irlanda, introduce un elemento muy diferenciador del resto de estos profesionales, que redunda y le confiere un perfil y una evolución lingüística particularmente interesante. La totalidad de las curtidurías que operaron en el siglo XVII y XVIII en el Señorío de Bizkaia, las cuales estaban integradas mayormente por irlandeses tal como se ha señalado anteriormente, se ubicaban en el entorno de las grandes villas, principalmente Bilbao y sus anteiglesias colindantes -Begoña, Abando y Arrigorriaga-, y, en mucha menor medida, Balmaseda y Durango. Esto implicaba para tales artesanos que, a buen seguro, en el interior de la tenería el lenguaje de trabajo sería el irlandés, y en el entorno comercial lo fuera el castellano, idioma que predominaba en la villa de Bilbao. Sin embargo, Guillermo Magra y su mujer María Morfil vivieron en Izkoa, un recóndito barrio de la anteiglesia de Mungia durante al menos diez años, un período en el que se infiere que lograron un considerable grado de integración en la comunidad que les rodeaba, conforme se extrae de las declaraciones que en su favor realizaron un buen número de sus vecinos en ciertos procesos judiciales, calificando a Magra de "quieto pacifico y no acostumbrado a ruido ni pendencia sino mui cuidadosso a su trabajo y oficio". Se constata, además, que todos los mencionados deponentes que conformaban su vecindario, además de declarar en su favor, lo hicieron en eus- 
kera por no saber la lengua castellana. ${ }^{29}$ Fruto de esta adaptación, y a pesar de no disponer de fuentes que lo corroboren, es muy factible pensar que en el tiempo que duró su estancia en la casería y barrio de Izkoa tanto Magra como su esposa acabaran hablando euskera en un entorno puramente vascoparlante, incluso albergando en su propia casa empleados que no conocían el castellano. Esta afirmación podría verse apoyada en el hecho de que años más tarde Magra realizó visitas y transacciones en el país vascofrancés (Iparralde) para ajustarse con los adjudicatarios de la explotación de las carnes de vacuno, de tal modo que le remitieran las pieles extraídas del sacrificio de los animales. Y análogamente en la provincia de Gipuzkoa, cerró contratos con proveedores de materias primas para sus talleres de curtido. Un aspecto que podría haber ayudado, y tal vez posicionado, a Guillermo en una situación ventajosa, es que desde su estancia en Mungia conocía muy probablemente el euskera, y en Gipuzkoa la población era mayoritariamente vascoparlante, al igual que en Baiona y Hasparren -ambas en Iparralde-, donde las clases populares empleaban igualmente este idioma (Sagarna 1984).

En este sentido, la evolución y exposición lingüística de Guillermo a lo largo de cuarenta años representa una carrera idiomática singular en el siglo XVIII. Nació en Adamstown (co. Wexford), ${ }^{30}$ y, a pesar de no tener constancia documental de ello, es muy probable que en un ámbito de lengua gaélica -con apellido de etnia Old Irish, integrado en una zona rural y en una baronía de mayoría irlando-parlante (Mernagh, 2008: 154)-. En mayor o menor medida, durante su infancia y adolescencia a comienzos del siglo XVIII -cuando la represión inglesa fue especialmente opresiva en Irlanda y las Penal Laws subyugaban la cultura autóctona-, acabaría hablando inglés. A su llegada a Bizkaia, y probablemente viviendo en el entorno de la villa de Bilbao, su lengua de acogida sería muy probablemente el castellano. Durante su estancia en Mungia, Guillermo acabaría inmerso en un universo vascoparlante, completando una progresión lingüística que por la variedad de los idiomas y por el contexto histórico en el que se produjo resulta sumamente interesante de estudiar. Desafortunadamente, las únicas vías de comunicación que se dispone de Guillermo Magra son escasas, todas ellas en castellano y siempre en documentos oficiales a través de las transcripciones llevadas a cabo por los escribanos.

\section{Conclusiones}

Es difícilmente discutible no considerar que las comunidades de inmigrantes, independientemente de la época y el lugar en el que se ubiquen, muestran una tendencia a buscar un equilibrio convivencial entre la sociedad receptora y la preservación de los rasgos de identidad propios 
de su tierra de origen, lo cual en cada caso se manifiesta de muy diferentes modos en función de factores múltiples, como el número de inmigrantes y su porcentaje sobre la población de acogida, la disposición de esta última a la convivencia con extraños, el marco socio-político en el que se produzca, las propias aspiraciones de los inmigrantes, y otros muchos. Y en este entramado social, la lengua nativa podría contemplarse como uno de los atributos identitarios más representativos y notorios. Es en este contexto en el que la comunidad de católicos irlandeses huidos de su isla por la ocupación y represión inglesa de los siglos XVII y XVIII se asentó en el Señorío de Bizkaia.

A pesar de que tal comunidad representaba una parte muy exigua sobre el total de la población vizcaína, ha quedado patente que tuvieron su presencia y relevancia en determinados ámbitos socio-económicos. Así pues, por extensión, cabe pensar que así lo hiciera su lengua, el gaélico irlandés. A pesar de que aún no se han llegado a encontrar documentos en tal idioma que acrediten su uso en Bizkaia, numerosos datos evidencian su existencia en el entorno social vizcaíno. Y aún más, su presencia se recoge en diversos espacios, a saber, los procesos de avecindamiento, las causas judiciales, las diligencias públicas y mercantiles de varios órganos de poder, etc. De este modo, puede justificarse que la lengua irlandesa tuvo un componente público en su paso por la Bizkaia moderna, principalmente ligado al comercio y las actividades portuarias de las villas y pueblos vizcaínos.

Análogamente, aunque más difícil de probar pero más intuitivamente aceptable, el gaélico encontraría su medio natural en el seno de la comunidad irlandesa instalada en Bizkaia, particularmente en los ambientes privados o socialmente menos expuestos, tales como la familia, las talleres de curtido de pieles con ocupación plenamente irlandesa, las congregaciones religiosas y, en general, las relaciones interpersonales que entre ellos pudieran darse, más aún, habiéndose comprobado que la cohesión social era una de las señas de identidad del colectivo.

A pesar de que el uso y presencia del irlandés en Bizkaia fueran tan exiguas, o al menos tanto como lo fue el impacto demográfico de sus componentes, no deja de ser menos relevante como fenómeno sociocultural en cuanto a la conservación de valores de una sociedad expulsada de su tierra. No deja de ser paradójico cómo un atributo cultural como es la lengua evoluciona de forma desigual en ámbitos sociales diferentes y no siempre relacionado con lo numeroso del grupo. Así, mientras que en Irlanda, bajo unas condiciones políticas represivas, el irlandés se fue diluyendo a lo largo de dos siglos hasta que, a finales del siglo XVIII y principios del siglo XIX, únicamente se hablaba en unos espacios muy limitados; por su parte, en una pequeña comunidad como la irlandesa de Bizkaia, el idioma se fue transmitiendo de generación en 
generación, al menos hasta que el colectivo se fue disolviendo, e incluso a finales del siglo XVIII, tal como se apunta anteriormente, se tiene constancia de su empleo entre los descendientes de irlandeses en Bizkaia, aunque muy probablemente no tuviera una gran implantación.

Ekain Cagigal Montalbán

Investigador independiente

48930 Getxo (Bizkaia)

ekaincagigal@yahoo.com

ORCID: 0000-0002-4300-199X

Recepción: 30/10/2018; Aceptación: 20/04/2019

\section{Notas}

1 Se hace preciso aclarar que el irlandés o gaélico irlandés, como lengua, pertenece a la familia de las lenguas indoeuropeas célticas, y más específicamente al grupo de las goidélicas, junto al gaélico escocés y al manés. Como simplificación, en lo sucesivo, se emplearán en el texto los términos "irlandés" o "gaélico" indistintamente, aludiendo siempre al gaélico irlandés, y obviando cualquier otra lengua céltica en el estudio.

2 En la bibliografía pueden encontrarse ciertas alusiones a la presencia de la lengua irlandesa en la Monarquía hispánica, pero de modo anecdótico o colateral; y, en todo caso, sin ser parte esencial del estudio (Martínez-Radío Garrido 2013: 164; Recio Morales 2006: 252-4; O’Scea 2002).

3 Archivo Histórico Foral de Bizkaia (en adelante, AHFB): Corregimiento, JCR0453/007, 33v-34r.

4 AHFB: Consulado, 0061/003, 30r.

5 AHFB: Consulado 0572/001/101.

6 AHFB: Corregimiento, JCR0451/024.

7 AHFB: Corregimiento, JCR1753/004.

8 AHFB: Corregimiento, JCR0373/004.

9 AHFB: Corregimiento, JCR1615/011.

10 AHFB: Alcalde de Bilbao, JCR0543/004.

${ }^{11}$ AHFB: Corregimiento, JCR0611/025.

${ }^{12}$ AHFB: Corregimiento, JCR1563/025.

${ }^{13}$ AHFB: Corregimiento, JCR1563/027.

${ }^{14}$ AHFB: Corregimiento, JCR1633/029, JCR1561/007.

${ }^{15}$ A modo de ejemplo, en 1750 Edmundo Kelly, subteniente graduado del ejército español, declaraba: “( ...) y que una noche le havia expresado dicho Nicolas [Dobli, irlandés residente en Bilbao] en una taverna hallandose con unos españoles (...)”. AHFB: Corregimiento, JCR3090/004.

${ }^{16}$ Un cálculo aproximado demostraría que la población irlandesa en Bizkaia durante los siglos XVII y XVIII no superaría, en el mejor de los casos, el 1\% de la población vizcaína total.

${ }^{17}$ A este respecto, resultaría de enorme interés un estudio comparativo del gaélico con el espacio socio-lingüístico en el que evolucionaron y coexistieron otros idiomas foráneos en el Señorío de Bizkaia, tales como el inglés o el francés, ahondando en la singularidad de que estos, a diferencia del primero, sí conservan constancia documental. De tal modo, se contempla y se propone esta vía de investigación para futuros trabajos.

18 A pesar de que ciertos historiadores discriminan entre los Old English y los anglonormandos, a los efectos del presente estudio y en la época estudiada, esta diferencia-

Lengua y migración / Language and Migration 12:1 (2020), 149-173

Edición impresa: ISSN 1889-5425. Edición en línea: ISSN 2660-7166. (C) Universidad de Alcalá 
ción no se considera relevante y se tratarán como un grupo étnico conjunto (O’Scea 2010: 122).

19 El mencionado estudio no recoge la totalidad de los irlandeses que se asentaron en Bizkaia, puesto que otros archivos han mostrado un mayor número de individuos irlandeses que los encontrados en el Archivo Histórico Eclesiástico de Bizkaia. Sin embargo, la muestra total, cercana a los 300 irlandeses censados, se considera suficientemente representativa para ser empleada como muestra estadística en los cálculos llevados a cabo.

20 A pesar de que la situación idiomática en Irlanda varía en función de los años, mapas de c. 1700 y c. 1800 , no se dispone de un detalle temporal del origen de los irlandeses de Bizkaia, de tal modo que para ambos años se realizará el contraste frente al agregado de datos del segundo grupo.

21 AHFB: Gobierno y Asuntos eclesiásticos, AJ03216/012, 1663,

22 AHFB: Bilbao Antigua, 0378/001/006, 1661, f. 260v.

23 AHFB: Gobierno y Asuntos eclesiásticos, AJ03226/008, 1690.

${ }^{24}$ AHFB: Gobierno y Asuntos eclesiásticos, AJ03211/019, 1652-3; AHFB: Gobierno y Asuntos eclesiásticos, AJ03221/011, 1674; AHFB: Bilbao Antigua, 0380/001/008; respectivamente.

25 Está documentado que los enlaces matrimoniales totalmente irlandeses se dieron con una elevada frecuencia en el caso de las parroquias vizcaínas, por encima del $30 \%$ de los matrimonios que involucraban algún irlandés. Este porcentaje es elevado si se considera que la ratio de hombres a mujeres irlandesas era aproximadamente de 2:1, de modo que casi todas las irlandesas casaron con compatriotas una vez en Bizkaia (Cagigal 2018).

${ }^{26}$ En fecha tan avanzada como 1790, cuando la lengua irlandesa ya atravesaba en Irlanda uno de sus momentos más decadentes, Juan Blas Doran y Tomas Moroni, nacidos en Bizkaia en la década de 1750, declaraban ser "ynteligente en la lengua yrlandesa" (AHFB: Corregimiento, JCR0209/024).

27 AHFB: Administración de Bizkaia, AJ00226/010.

$28 \mathrm{O}$ William McGragh en su forma inglesa, o Liam Mac Craith en su forma gaélica.

${ }^{29}$ AHFB: Corregimiento, JCR0619/015.

30 AHFB: Genealogías, Reg. 43, Gen. 669.

\section{Referencias bibliográficas}

Bernal Serna, Luis María. 2003. "Los espacios de la violencia. Tabernas y fiestas en Vizcaya (1560-1808)". Vasconia: Cuadernos de historia - geografía, 33. 409-424.

Bernal Serna, Luis María. 2011. "Contenidos principales y conclusiones de la tesis doctoral Crimen y violencia en la sociedad vizcaína del Antiguo Régimen (1550-1808)". Clio E Crimen: Revista del Centro de Historia del Crimen de Durango, 8. 481-522.

Bilbao Acedos, Amaia. 1999. "Los irlandeses y el sector del curtido en Bizkaia en el siglo XVIII". Bidebarrieta. Anuario de Humanidades y Ciencias Sociales de Bilbao, 4. 295-309.

Bilbao Acedos, Amaia. 2004. Los irlandeses de Bizkaia: "los chiguiris", siglo XVIII, Bilbao: Fundación Bilbao Bizkaia Kutxa.

Boone, Marc, y Heleni Porfyriou. 2007. "Introduction to Part II”. En Cultural Exchange in Early Modern Europe 1400-1700, Donatella Calabi y Stephen Turk Christensen (eds.), Vol. II, Cambridge: Cambridge University Press. 59-64.

Burke, Peter. 2004. Languages and Communities in Early Modern Europe. Cambridge: Cambridge University Press.

Cabantous, Alain. 1990. Le Ciel dans la mer. Christianisme et civilisation maritime (XVIeXIXe siècle). Paris: Fayard. 
Cagigal, Ekain. 2018. "Irish migration to early modern Biscay”, Archivium Hibernicum, 71. 158-174.

Cifuentes Pazos, José Manuel. 2003. “El clero de Bilbao en el Antiguo Régimen: número, procedencia geográfica y extracción social”. Bidebarrieta. Anuario de humanidades y ciencias sociales de Bilbao. 12. 278-302.

Coolahan, Marie-Louise. 2010. Women, Writing, and Language in Early Modern Ireland. Oxford: Oxford University Press.

De Fréine, Seán. 1978. The Great Silence: The Study of a Relationship Between Language and Nationality. Minneapolis: Irish Books \& Media.

Doyle, Aidan. 2015. A History of the Irish Language: From the Norman Invasion to Independence. Oxford: Oxford University Press.

FitzGerald, Garret. 1984. "Estimates for baronies of minimum level of Irish-speaking amongst successive decennial cohorts: 1771-1781 to 1861-1871”. Proceedings of the Royal Irish Academy: Archaeology, Culture, History, Literature, 84C. 117-155.

Grande Pascual, Andrea. 2015. "Violencia interpersonal en la sociedad vizcaína a finales de Antiguo Régimen”. Clío E Crímen: Revista del Centro de Historia del Crimen de Durango, 12. 215-232.

Guiard Larrauri, Teófilo. 1972. Historia del Consulado y Casa de Contratación de la villa de Bilbao. Bilbao: Editorial La Gran Enciclopedia Vasca.

Hindley, Reg. 1990. The Death of the Irish Language: A Qualified Obituary. Oxon: Routledge.

Keegan, Kevin. 2013. “The Linguistic Geography of the Contact Zone: The Complementarity of Orality and Literacy in Colonial Ireland", Historical Geography, 41. 80-93.

Labayru, Estanislao Jaime. 1971. Historia General del Señorío de Bizcaya. Tomo V. Bilbao: Biblioteca de la Gran Enciclopedia Vasca.

Mac Giolla Chríost, Diarmait. 2004. The Irish Language in Ireland: From Goidel to Globalisation. Oxon: Routledge.

Martínez-Radío Garrido, Evaristo C. 2013. "Herederos de Irlanda al servicio de España durante la Guerra de la Independencia: el caso del primer batallón del Regimiento Hibernia”. Trocadero, 25. 161-185.

Mernagh, Michael. 2008. "A Brief History of Languages in County Wexford. As we used to say". The Past: The Organ of the Ui Cinsealaig, 29. 145-164.

Ó Cuiv, Brian. 1969. A View of the Irish Language. Dublin: Stationery Office.

Ó Cuív, Brian. 1976. “The Irish language in the Early modern period”. En Early Modern Ireland, 1534-1691, Theodore William Moody, Francis Xavier Martin, Francis John Byrne (eds.). Oxford: Clarendon Press. 509-545.

Ó Gráda, Cormac. 2013. “Cé Fada le Fán”. Dublin Review of Books, 34.

O'Connor, Thomas. 2017. "The domestic and international roles of Irish overseas colleges, 1590-1800". En College Communities Abroad: Education, Migration and Catholicism in Early Modern Europe, Liam Chambers y Thomas O'Connor (eds.). Oxford: Oxford University Press. 90-114.

O’Néill, Diarmuid. 2005. Rebuilding the Celtic Languages: Reversing Language Shift in the Celtic Countries. Ceredigion: Y Lolfa.

O’Scea, Ciaran. 2002. “En Busca de Papeles. La transformación de la cultura oral de los inmigrantes irlandeses desde La Coruña hasta la Corte”. En Irlanda y la Monarquía Hispánica: Kinsale 1601-2001. Guerra, política, exilio y religión, Óscar Recio Morales, Bernardo José García García, Miguel Angel de Bunes Ibarra, Enrique García Hernán (eds.). Madrid: CSIC. 359-380.

O’Scea, Ciaran. 2010. “Nominación de los irlandeses en España, rechazo y asimilación (1600-1680)”. En Gregorio Salinero, Isabel Testón Núñez (eds.): Un juego de engaños: movilidad, nombres y apellidos en los siglos XV a XVIII. Madrid: Casa de Velázquez. 121-138.

Ravenstein, Ernst Georg. 1879. “On the Celtic Languages in the British Isles: A Statistical Survey”. Journal of the Statistical Survey, 42. 579-636.

Lengua y migración / Language and Migration 12:1 (2020), 149-173

Edición impresa: ISSN 1889-5425. Edición en línea: ISSN 2660-7166. @ Universidad de Alcalá 
Recio Morales, Óscar. 2002. "De nación irlandés”: percepciones socio-culturales y respuestas políticas sobre Irlanda y la comunidad irlandesa en la España del XVII”. En Irlanda y la Monarquia Hispánica: Kinsale 1601-2001. Guerra, politica, exilio y religión, Óscar Recio Morales, Bernardo José García García, Miguel Angel de Bunes Ibarra, Enrique García Hernán (eds.). Madrid: CSIC. 315-340.

Recio Morales, Óscar. 2006. "Irish émigré group strategies of survival, adaptation and integration in seventeenth- and eighteenth-century Spain”. En Irish Communities in Early-Modern Europe, Thomas O’Connor y Mary Anne Lyons (eds.). Dublin: Four Court Press. 240-266.

Sagarna, Andoni. 1984. “Euskara XVIII. mendean”. Cuadernos de Sección. Hizkuntza eta Literatura, 3. 17-114.

Santoyo, Julio César. 2008. “William Frankland, autor 'anónimo' de An Account of Saint Sebastian (Londres, 1700)". Sancho el sabio: Revista de cultura e investigación vasca, 29. 49-60.

Smyth, William J. 2006. Map-making, Landscapes and Memory: A Geography of Colonial and Early Modern Ireland, c.1530-1750. Cork: Cork University Press.

Zabala Uriarte, Aingeru. 1994. Mundo urbano y actividad mercantil, Bilbao 1700-1810. Bilbao: Fundación Bilbao Bizkaia Kutxa.

Lengua y migración / Language and Migration 12:1 (2020), 149-173 Edición impresa: ISSN 1889-5425. Edición en línea: ISSN 2660-7166. @ Universidad de Alcalá 